\title{
A Review of the Actual Knowledge of the Processes Governing Growth and Development of Long Bones
}

\author{
Ugo Ernesto Pazzaglia, ${ }^{1}$ Giampiero Beluffi, ${ }^{2}$ Anna Benetti, ${ }^{3}$ Maria Pia \\ Bondioni, $^{4}$ and Guido Zarattini ${ }^{1}$
}

${ }^{1}$ Orthopaedic Clinic, University of Brescia, Brescia, Italy; ${ }^{2}$ Department of Pediatric Radiology, San Matteo General Hospital, Pavia, Italy; ${ }^{3}$ Department of Pathological Anatomy, University of Brescia, Brescia, Italy; ${ }^{4}$ Department of Pediatric Radiology, University of Brescia, Brescia, Italy

\begin{abstract}
Autoptic samples of human bones (from 8 weeks of gestation to 12 years of age) and a second group of serial, skeletal x-rays (required for pathologies not related to bone dysplasia in children from 4 months to 17 years of age) provided the material for the analysis of the physes normal growth mechanism presented in this review. Before the appearance of the ossification centers epiphyseal growth rests exclusively on chondrocytes proliferation (interstitial growth), without any detectable differentiated cellular organization. When endochondral ossification starts a defined spatial disposition of chondrocytes and a corresponding organization of the intercellular matrix is set up, so that it is possible to identify a growth vector corresponding to the columns of piled chondrocytes with direction from hypertrophic toward the proliferative cell layers. The complexity of the tubular bones growth process is well represented by the spatial arrangement of the growth vectors. In the late epiphyseal growth another mechanism is active in addition to endochondral ossification, namely, articular cartilage interstitial growth and subchondral remodelling. The knowledge of the normal mode of organization of the physis and its temporal sequence can help to better understand of the deviaton from the normal development of metaphyseal and epiphyseal dysplasias.
\end{abstract}

Keywords bone growth, endochondral ossification, epiphysis, epiphyseal dysplasia

\section{INTRODUCTION}

The classification of ostechondral dysplasias is founded on a well-established tradition of the radiographic study of the skeleton, and specific types of disorder in individual cases are referred to in broad terms to the anatomic division of growing bones into epiphysis, metaphysic, and diaphysis. Pathologic limitations to a single compartment, however, are uncommon, and for accepted custom the defect is referred to the component or components predominantly affected (1).

Different combinations of shape deformities, growth defects, and abnormalities of calcification/ossification processes have contributed to identification of specific disorders: the spectrum has been further broadened with the identification of extraskeletal tissues and organ involvement, modality of transmission, and when possible, genetic defect [2-4].

Even though the most recent advances in knowledge and systematization of skeletal dysplasias are considerable, a wide margin of uncertainty remains to be defined 
of many specific disorders presenting in individual cases of a wide variability of phenotipic expression. Among these epiphyso-metaphyseal dysplasias are the most evident example of a complex and diversified spectrum of cartilagineous disorders, whose diagnostic criteria still rest on clinical and imaging assessment [5, 6], enriched by the most recent magnetic resonance technology [7-10]. The definition of the growth vectors on histologic slides documenting the normal physeal development, associated with the findings of traditional x-rays, can provide a hint to integrate imaging data in the light of the actual knowledge of normal and pathologic shape development of the long bones cartilage models.

\section{MATERIALS AND METHODS}

The observations presented in this review were originated from the histologic slides made available to the senior author (UEP): a) Four autoptic cases of children between 6 months and 12 years who died from diseases not involving the physeal structure from the historical collection of the Morbid Anatomy Department of the Royal National Orthopaedic Hospital of London (thanks to the courtesy of PD Byers); of these cases were known sex, age, diagnosis, and the available proximal half of a long bone (humerus or femur in all four and also a tract of the lumbar spine in two). b) Twenty-five stillborn fetuses from 8 weeks of gestational age to term from an historical collection whose vertebral columns were used in 1989 to study the notochordal remnants in the human intervertebral disk [11] (thanks to the courtesy of JR Salisbury and PD Byers); of these fetuses was known only the length.

All the bone specimens had been fixed in formaline: most of them had been decalcified in ethylenediaminetetraacetic acid or in a formic acid solution, embedded in paraffin and stained with H\&E. Few of them were embedded undecalcified in methylmetacrylate resin, and the sections cut with a motorized heavy microtome had been stained with solochrome or with the Von Kossa method and counterstained with neutral red.

The x-rays documentation of the physes normal development were obtained from subjects different from those of the histology: from the files of the Pediatric Radiology Department, University of Brescia. Ten children between the ages of 4 months to 17 years were selected who had serial x-ray controls performed once a year for at least 5 years because of pathologies not related to bone dysplasia. These x-rays included an antero-posterior projection of the pelvis and/or lower limbs. The histology and x-ray imaging were used to illustrate the review.

\section{OBSERVATIONS}

\section{Prenatal and early childhood bone growth}

The ossification centers development inside the cartilage model of long bones has been extensively investigated in animal experimental models [12-17] and these observations can be extrapolated to the growth and development of human bones. However, a systemic and controlled analysis of the latter is not possible for obvious ethical reasons; therefore, only a discontinuous documentation of the normal bone growth could be collected.

In the early gestational age the first ossification centers in long bones cartilage model appear at level of the diaphysis when the physes were still completely formed by cartilage, but already conforming to the final shape of the proximal or distal end of the bone. Chondrocytes of physes are small and evenly distributed inside the matrix; their proliferation and the matrix synthesis accounts for the size increments during all 
the pre-ossification phases (either ante- or post-natal), but without any appreciable change of shape. This type of growth (interstitial) is evenly distributed in the context of the physis and does not imply a differentiated cellular organization. It escapes $\mathrm{x}$ ray observation but can be ascertained by echograms or MR. Epiphyseal ossification of the major long bones of upper and lower limbs follows a temporal sequence [18, 19] characterized by a series of transformations at the cellular level, which do not differ from those of the diaphyseal ossification center and are known as "endochondral ossification." They include:

1. proliferation, enlargement, and lining up of chondrocytes

2. hypertrophy of chondrocytes

3. $\mathrm{CaPO}_{4}$ deposition on the matrix interposed between hypertrophic chondrocytes

4. apoptosis of hypertrophic chondrocytes and vessels penetration between calcified cartilage septa

5. differentiation of osteoblasts and deposition of bone matrix on the calcified cartilage matrix (primary bone trabeculae)

6. remodeling of trabeculae from primary to secondary (in other words, only lamellar without a core of calcified cartilage).

The diaphyseal ossification center early in the fetal development is remodelled progressively and substituted by the periosteal lamellar bone. The whole physis is cartilagineous with a differentation toward the diaphysis corresponding to the metaphyseal growth plate. Its shape does not correspond to the external shape of the epiphysis. The typical cellular organization of the endochondral ossification and the chondrocytes columns are oriented parallel to the bone major axis at the top of the ossified diaphysis and below the epiphysis (still completely cartilagineous).

The epiphyseal ossification centers appear later and show the same chondrocytes maturative pattern as in the metaphyseal growth plate cartilage. However, here the three-dimensional architecture is spherical rather than bi-planar. This spatial arrangement is not usually appreciated in histologic slides because only a few of the chondrocyte columns lay in the plane of the section and the oblique planes of the other columns give the false impression of a disorderly arranged ossification with a vague resemblance to that of the metaphyseal growth plate (Figures 1a-1c). From these histologic images, it was deduced that in the epiphyseal ossification center the layer of growing cartilage extended on the whole surface of a ball and that its shape did not correspond to the external shape of the epiphysis.

The endochondral ossification of the epiphysis was also surveyed by sequential $\mathrm{x}$-rays and in the early phases of its development presented remarkably similar features in all epiphyses. It was placed in the center of the epiphysis (or eccentric if more ossification centers pertained to that epiphysis development) and had a rounded or slightly oval shape.

From the time of appearance of the ossification center, the physeal growth is governed by the interstitial chondrocytes proliferation and by the endochondral ossification advancement from the center to the periphery [20-23]. The progressive occupation of the epiphyseal volume by the ossification center (Figures 2a-2d) showed that the rate of advancement of the endochondral ossification is faster than expansion driven by the cartilage interstitial growth.

As illustrated by our histology and well documented in literature [24, 25], the endochondral growth is sustained by a well defined geometrical asset of chondrocytes. The active site of dimensional growth is located in the germinative cells layer and the process could be represented by a vector parallel to the cartilage columns and directed 


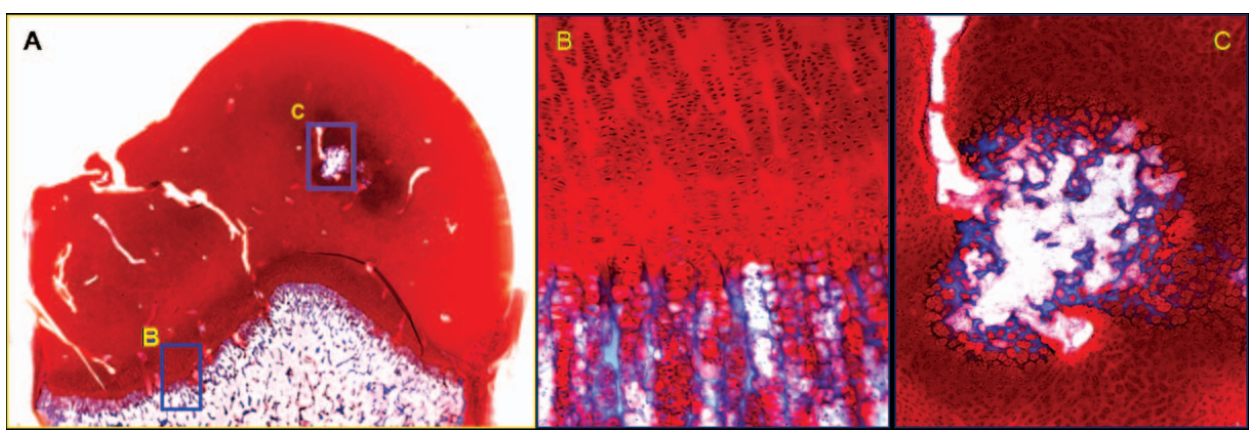

FIGURE 1 (A) (Solochrome, 1,5 $\times$ ) Normal aspect of the humeral proximal physis at age 6 months showing the first appearence of the primary epiphyseal ossification center (box C) and the metaphyseal growth plate cartilage (box B). (B) (Solochrome, 100×) Detail of the metaphyseal growth plate showing the columns of chondrocytes and the primary methaphyseal trabeculae of the growth plate, parallelely oriented to the bone major axis (biplanar asset). (C) (Solochrome, 40×) Detail of the epiphyseal ossification center showing the spatial organization of chondrocytes and primary trabeculae of the epiphyseal ossification center: they reproduce the same chondrocytes maturative pattern but the geometrical-asset is multiplanar. (box $\mathrm{C}$ ).

from the hypertrophic toward the germinative cells layer (Figure 3). Considering a whole long bone, the three-dimensional distribution of these vectors could be represented schematically as shown in the figure: it is evident that along the longitudinal axis, the vectors of the metaphyseal growth plate cartilage and those of the epiphyseal ossification center on the same line have opposite directions, like if the apiphysis would be pushed up and the diaphysis pushed down. In terms of the growth mechanics of the whole system, the middle compartment (diaphysis) is the stable point, and expansion of the cartilages (longitudinal growth) occurs at the extremities because the vectors of the distal physis are inverted in respect to the proximal.

\section{Late childhood and youth bone growth}

In the structured bone epiphysis, the peripheral expansion of the ossification center (Figure 4) ceases when the proliferative cells layer chondrocytes have reached the perichondrium (now periosteum) or, on the joint front, the level corresponding to the subchondral bone [26]. The end of this phase of growth is characterized on x-rays by a shape of the epiphysis which conforms to that of the mature bone epiphysis (Figure 5). The figure documents the growth and shape modulation of the proximal, femoral epiphysis; however, a similar evolution (but at different ages) can be observed

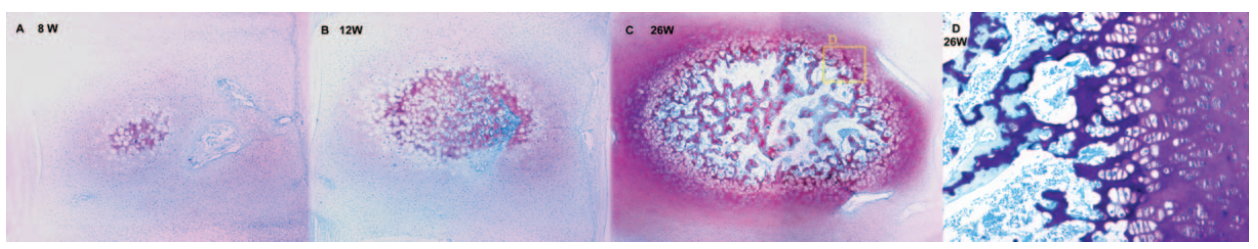

FIGURE 2 (H\&E, 40 $\times)$ The development of the ossification center of the human vertebral body in pre-term spine (A): age 8 weeks, showing hypertrophy of chondrocytes and calcification of the interposed cartilage septa. (B) Age 12 weeks showing primary trabeculae organization with a welldefined spatial arrangement. (C) Age 26 weeks showing the further expansion of the ossification center with its trabecular architecture: most trabeculae still show the cartilage core. (D) Age 26 weeks $(100 \times)$, showing the typical endochondral ossification pattern not different from that of the long bones metaphyseal growth plate cartilage. 

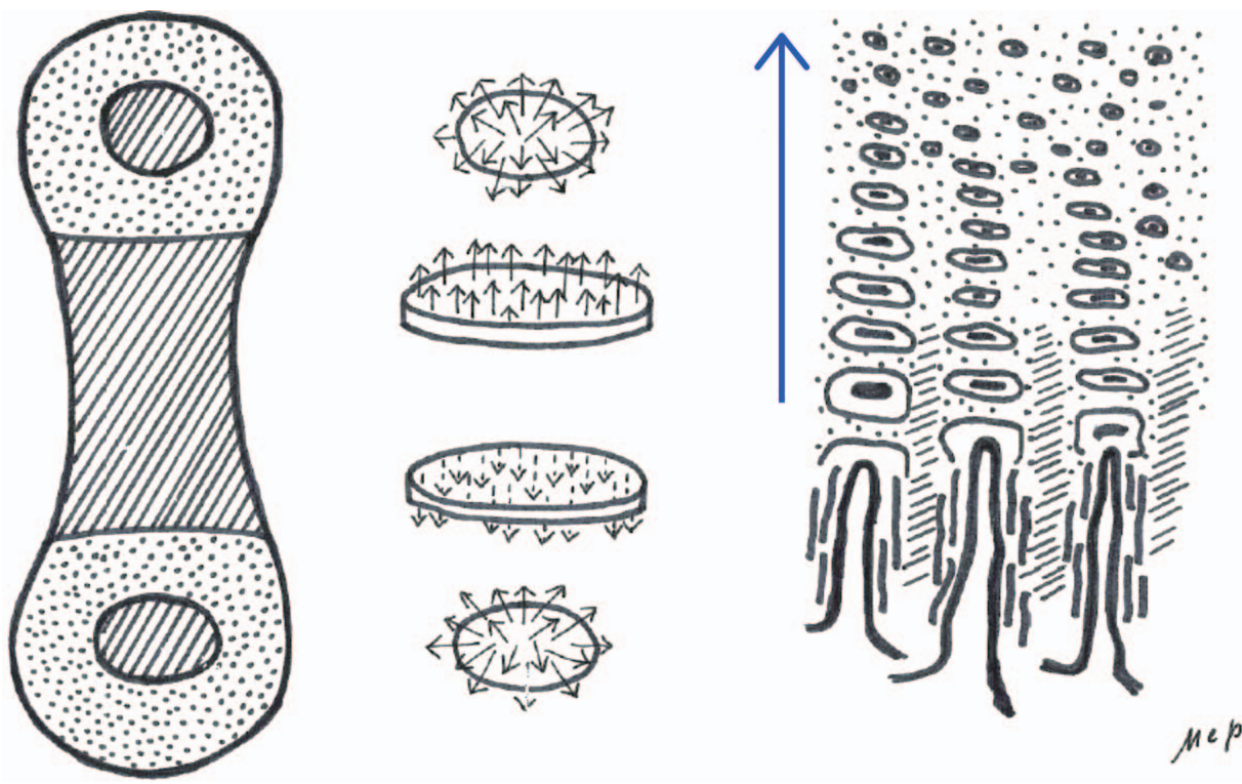

FIGURE 3 Growth vector definition and its variable orientation in the growth of proximal and distal metaphyseal growth plates and in the epiphyseal ossification centers of long bones.

in the other, large epiphyses of lower limbs bones. From this point onward, the only structure with a typical maturative and columnar pattern of chondrocytes is the metaphyseal growth plate cartilage. Further size increments and shape moulding of the epiphyses are observed in both longitudinal and latitudinal directions (Figure 6). However, this growth is governed by other mechanisms: the interstitial chondrocytes proliferation at the level of the articular cartilage and the apposition/resorption modeling at the level of the subchondral bone (Figure 7).

The circumpherential enlargement of the diaphysis is implemented by the periosteum with the sequential apposition of lamellar bone layers (Figure 8).

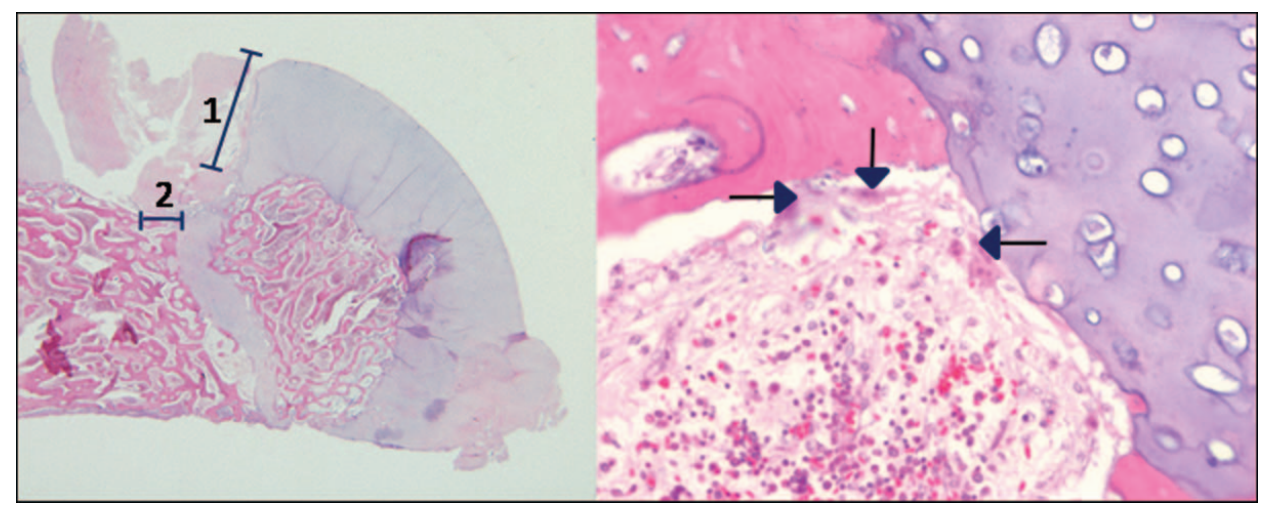

FIGURE 4 Mechanism of growth of the femur proximal epiphysis approximatively from age 5 years (change of shape of the ossification center from spherical to frustum of sphere): (A) H\&E, $1,5 \times$ ) coronal section of the femur proximal physis at 5 years, when the peripheral layer of cartilage (bar 1) is still thicker than the metaphyseal growth plate (bar 2). Detail of (B) at $200 \times$ showing the remodelling of the subchondral bone carried out by osteoclasts (arrows) and absence of the columnar pattern of chondrocytes. 


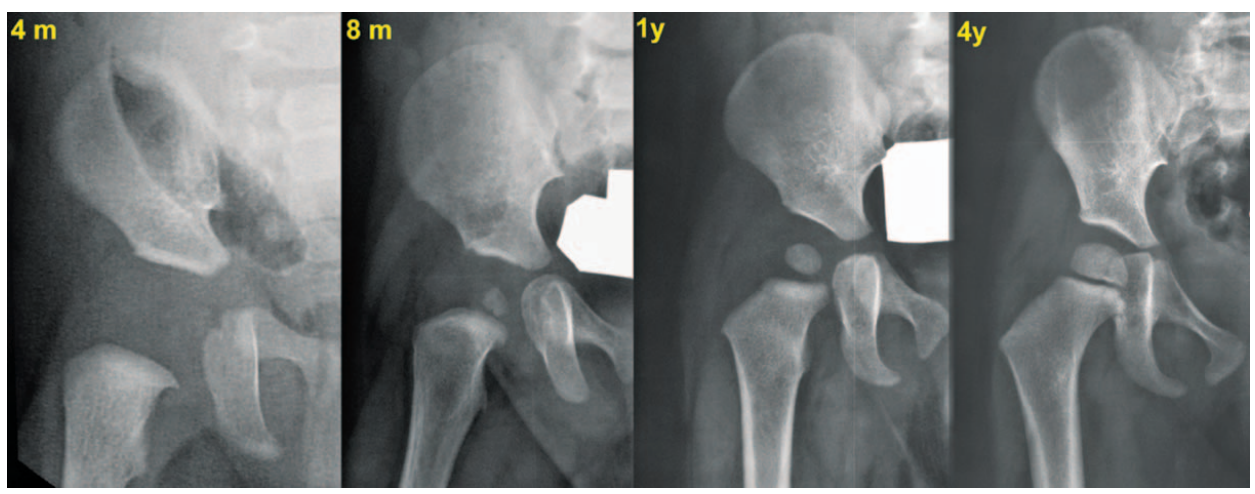

FIGURE 5 Radiographic aspects of the expansion of the proximal femur ossification center, which maintains the spherical shape from age 8 months to 4 years: (A) age 4 months; (B) age 8 months; (C) age 1 year; (D) age 4 years.

\section{DISCUSSION}

In normal bone development, it is possible to observe different modes of cells aggregation inside the cartilage model, which appears to determine the size increments and the shape modulation of the growing bone.

Before ossification starts, the cartilage model increases its size through chondrocyte proliferation and pericellular matrix synthesis; this type of growth is known as "interstitial" [27]: if the density of the cells remain evenly distributed, the shape of the model cannot change but only its volume increases: on the contrary, a differentiated topography of chondrocytes proliferation can modulate shape variations. This simple pattern of growth is present in the earlier phases of development when mesenchymal condensation in the limb buds of 14-day-old embryos forms the cartilage anlage of the appendicular skeleton long bones [15]. However, this interstitial growth, even if at a slower rate, remains active for the whole growth period as documented by the articular cartilage expansion when the epiphysis presents with its definitive shape, but further size increment is going on.

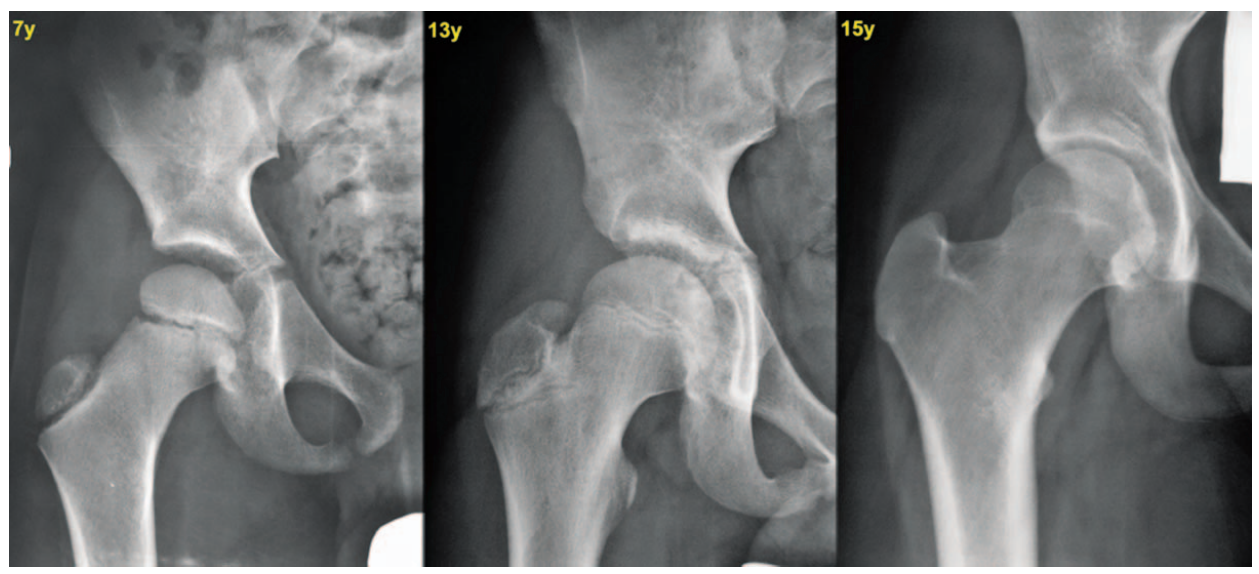

FIGURE 6 Radiographic aspect of the shape changes of the proximal femur ossification center in later growth: the shape is conformed to a frustum of sphere from age 7 years to 15 years: (A) age 7 years; (B) age 13 years; (C) age 15 years. 


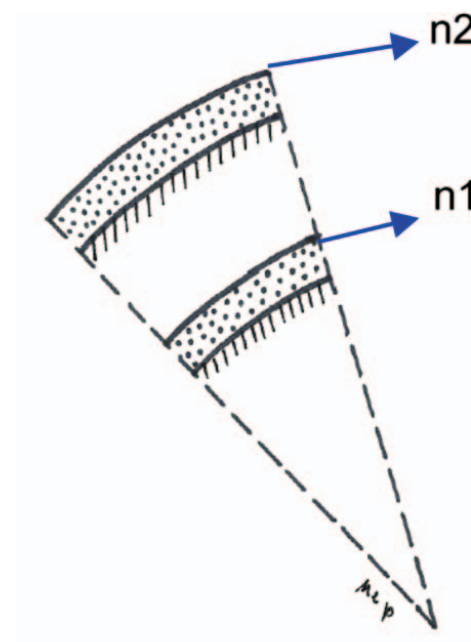

C

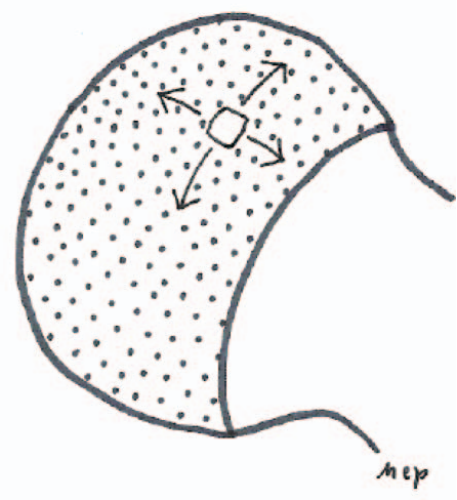

$\because \because$ ARTICULAR

C FEMURAL HEAD CENTER

$\mathbf{r} 1=$ HEAD RAY AT TIME $\mathbf{t} ; \mathbf{r} \mathbf{2}=$ HEAD RAY AT TIME 2

r2-r1 = HEAD GROWTH

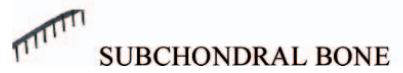

FIGURE 7 Pattern of the proximal femur epiphysis growth in late adolescence: the articular cartilage interstitial growth and the subchondral bone remodelling must cope with the enlargement of the head site.

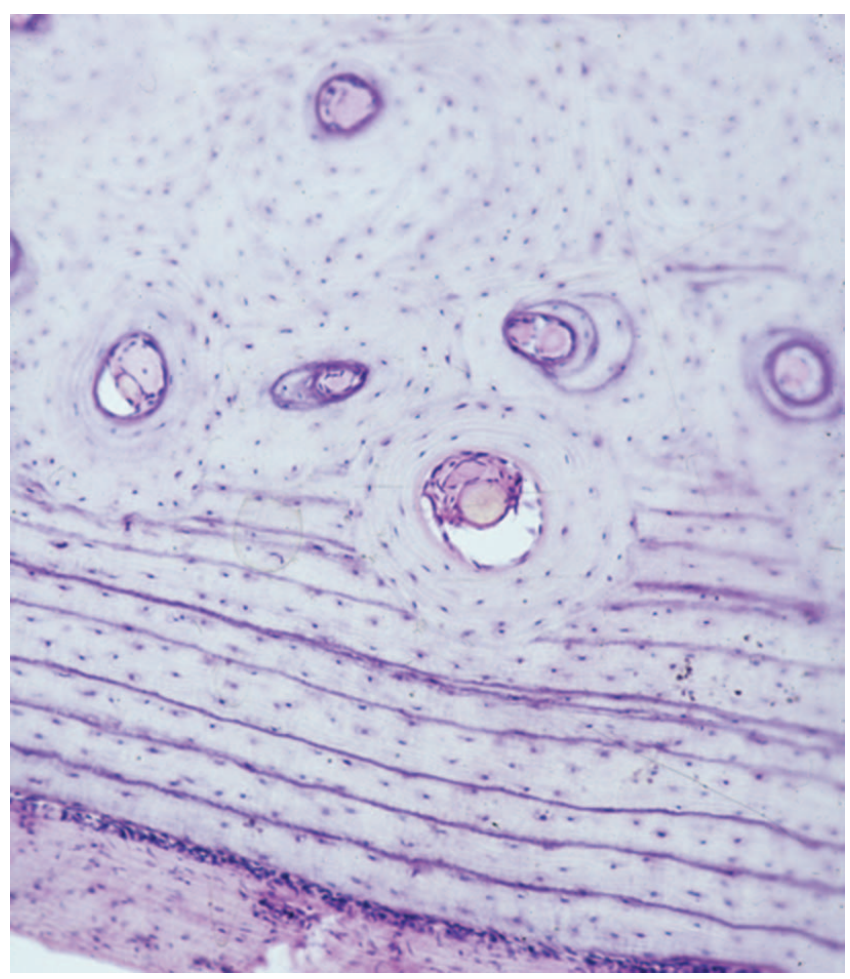

FIGURE 8 (H\&E, 100 $\times$ ) Lamellae apposed to the periphery of the diaphysis by the periosteum and haversian remodelling progressing from the inside of the cortex illustrating the mechanism of circumpherential enlargement of this bone compartment. 


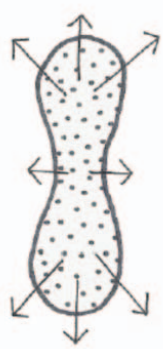

BONE
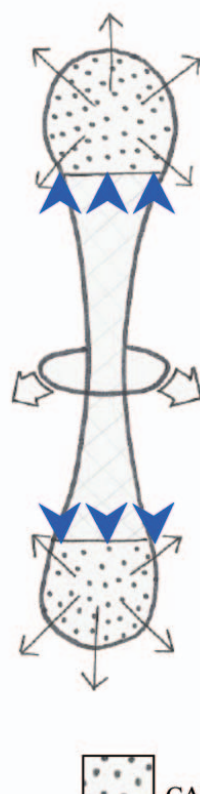
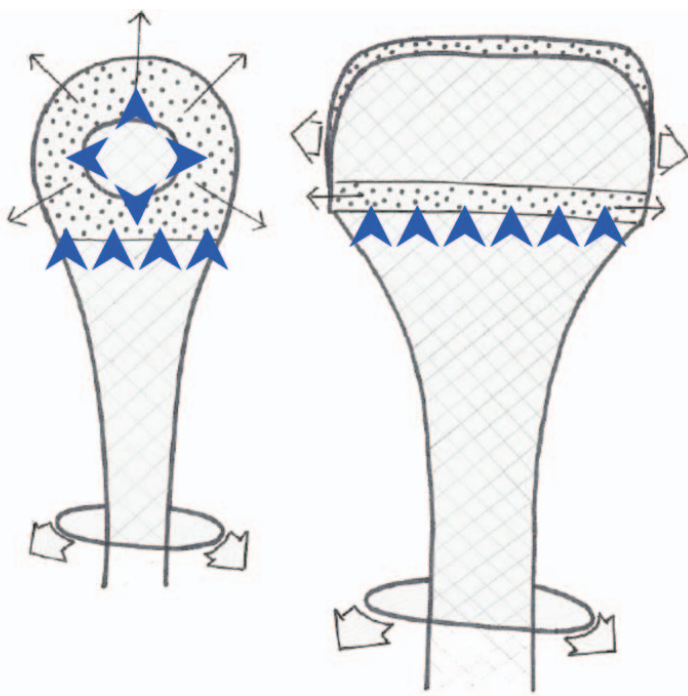

$\mu e p$

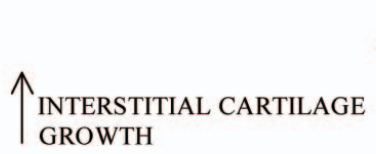

CARTILAGE GROWTH

ENDOSTEAL OSSIFICATION GROWTH

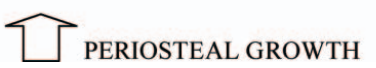

FIGURE 9 Skeme representing the different mechanisms of bone growth from the earlier cartilage model to the definitive shape.

The other growth controlling mechanism is always characterized by the same two elemental phenomena-cell duplication and matrix production-but ordered in a more complex plan where other changes in the matrix intervene, like $\mathrm{CaPO}_{4}$ deposition, neoangiogenesis, and activation of other cellular types like osteoblasts and osteoclasts. Moreover, all these phenomena appear strictly oriented along a direction, in such a way that growth can be represented by a vector.

The volume increase of chondrocyte (hypertrophy), which characterizes the ossification center of the epiphysis and the ordered, columnar arrangement of the same cells in the metaphyseal growth plate, has been for a long time recognized as the fundamental structural organization controlling the bone longitudinal growth $[23,28,29$, 30]. Less attention has been paid to the epiphyseal ossification, which starts later with a spherical center and presents with the same pattern of chondrocytes piling, but with a three-dimensional asset. Its role is controversial: it has been suggested that the contribution to growth of the epiphyseal ossification center is limited to the peripheral expansion [31, 32]. However, the architecture and analysis of the growth vectors, documented here, support the hypothesis that the epiphyseal ossification takes part in some phases of the bone development to the general length increment with two fronts: one facing the metaphyseal growth plate but with the growth vector in the opposite direction, the other facing the joint surface with the vector in the same direction as that of the growth plate.

In a later phase of the bone development, the osteoblasts apposition and the subchondral modelling associated with interstitial growth of the articular cartilage are the only cellular mechanisms responsible for the size increments of the epiphysis, as well as the periosteal apposition controls the outer diaphyseal enlargement (Figure 9). 
It is possible to establish, with a rough degree of accuracy, a correlation between these patterns of organization at a histologic level and the radiographic aspects documented by x-rays during the time interval of single bones development. While the diaphyseal ossification process takes place at a gestational age when x-rays examination is not applicable, the epiphyseal ossification does it, because it starts in the last prenatal stage and later after delivery. The first opacities in the middle of the cartilagineous epiphysis correspond to the calcium salt deposition on the cartilage matrix between hypertrophic chondrocytes. When the same becomes enlarged and assumes a rounded contour, it corresponds to the formation of the first, structured center with primary and secondary bone trabeculae.

It has been possible to compile a sufficiently accurate x-ray atlas with the time of appearance of the epiphyseal nuclei of wrist and hand [18] or of the other ossification centers distributed in the skeleton [19] and on these is founded the ascertainment of the skeletal age. Its limit of accuracy depends on the sequence and regularity of the cellular processes which have been illustrated earlier and on the range of variation of the normal development.

The x-rays imaging of the epiphyseal ossification was found to be also a rough index of the developmental phase when the maturative pattern of chondrocytes and their columnar arrangement disappear. The end of this phase can be approximately identified on bone x-rays when the epiphyseal ossification center looses its spherical shape and becomes more conformed to the final shape of the bony epiphysis.

In the lower limb main bone epiphyses, the loss of the columnar arrangement and the maturative pattern of chondrocytes of the epiphyseal ossification center occurred between 4 and 7 years of age and was particularly evident on the flattened front of the ossification center facing the metaphyseal growth plate cartilage. The age of disappearance of the cartilage cells asset in serial columns of the epiphyseal ossification center showed a remarkable variability, which has been observed to depend upon the individual bone considered and the sex and the racial differences of the bone development [19].

The histologic and serial x-ray study of the epiphyseal growth in the interval between the ages of 8 and 10 years and the skeletal maturity gave evidence of a further longitudinal and latitudinal increase of epiphyseal diameters, which cannot be explained by endochondral ossification. The recognized proliferative activity of cells at the level of the perichondrial groove of Ranvier [33] supports the latitudinal expansion of the metaphyseal growth plate, but do not explain the contemporary enlargement of the dome of the epiphyses, which are now completely ossified. The mechanism of the epiphyses growth in this phase is driven by the subchondral remodelling and interstital growth of the articular cartilage.

Osteochondral dysplasias present with varying disturbances of cartilage growth and organization, most often in defect but also, even if less frequently, with overor/and asymmetrical growth of individual physes [3, 4, 34-36]. They should be better refereed to the phases of the physeal growth and development rather than to the simple anatomic distinction between epiphysis and metaphysis.

\section{Declaration of Interest}

The authors report no conflicts of interest. The authors alone are responsible for the content and writing of the paper.

\section{REFERENCES}

[1] Silverman FN. Reflections on epiphyseal dysplasias. Am J Rad 167:835-842, 1996.

[2] Taybi H, Lachman S. Radiology of Syndromes, Metabolic Disorder and Skeletal Dysplasias, 4th ed. St. Louis, MO: Mosby, 1996. 
[3] Spranger JW, Brill PW, Poznanski AK. Bone Dysplasias. An Atlas of Genetic Disorder of Skeletal Development, 2nd ed. Oxford: Oxford University Press, 2002.

[4] Castriota-Scanderberg A, Dalla Piccola B. Abnormal Skeletal Phenotypes from Simple Signs to Complex Diagnosis. Berlin-Heidelberg: Springer Verlag, 2005.

[5] Silverman FN. Dysplasies epiphysaries: Entité protéiforme. Ann Radiol 4:833-867, 1961.

[6] Silverman FN. Dysplasia epiphysealis hemimelica. Seminars in Roentgenol 24:246-258, 1989.

[7] Oestreich AE, Prenger EC. MR demostrates cartilagineous megaepiphyses of the hips in Kniest dysplasia of the young child. Pediatr Radiol 22:302-303, 1992.

[8] Iwasawa T, Aida N, Kobayashi N, Nishimura G. MRI findings of dysplasia epiphysealis hemimelica. Pediatr Radiol 26:65-67, 1996.

[9] Lang IM, Azouz EM. MRI appearances of dysplasia epiphysealis hemimelica of the knee. Skeletal Radiol 26:226-229, 1997.

[10] Peduto AJ, Frawley KJ, Bellemore MC, Kuo RS, Foster SL, Onikul E. MR imaging of dysplasia epiphysealis hemimelica: bone and soft tissue abnormalities. Am J Roentgen 172:819-823, 1999.

[11] Pazzaglia UE, Salisbury JR, Byers PD. Development and involution of the notochord in the human spine. J Roy Soc Med 28:413-415, 1989.

[12] Tickle C, Eichele G. Vertebrate limb development. Ann Rev Cell Biol 10:121-152, 1994.

[13] Speer DP. Collagenous architecture of the growth plate and perichondrial ossification groove. J Bone J Surg 64A:399-407, 1982.

[14] Floyd WE 3rd, Zacleske DJ, Schiller AL, Trahiu C, Nankin HJ. Vascular events associated with the appearance of the secondary center of ossification in the murine distal femur epiphysis. $J$ Bone $J$ Surg 69A:185-190, 1987.

[15] Hannes RW. The evolution of epiphyses and of endochondral bone. Biol Rev 17:267-91, 1942.

[16] Masond I, Shapiro F, Moses A. Tibial epiphyseal development: a cross-sectional histologic and histomorphometric study in the New Zealand white rabbit. J Orthop Res 4:212-20, 1986

[17] Heickel HVA. On ossification and growth of certain bones of the rabbit: with a comparison of the skeletal age in the rabbit and in man. Acta Orthop Scand 29:171-84, 1959.

[18] Greulich WW, Pyle SI. Radiographic Atlas of Skeletal Development of the Hand and Wrist, 2nd ed. Stanford CA: Stanford University Press, 1959.

[19] Garn SM, Rohmann CA, Silverman FC. Radiographics standards of postnatal ossification and tooth classification. Med Radiography and Photography 43:45-66, 1967.

[20] Salomon L. Diametric growth of the epiphyseal plate. J Bone J Surg 48B:170-177, 1966.

[21] Hert J. Growth of the epiphyseal plate in circumference. Acta Anat 82:420-436, 1972.

[22] Lutfi AN. The role of cartilage in long bones growth. J Anat 117:413-417, 1974.

[23] Kember NF, Sissons HA. Quantitative histology of the human growth plate. J Bone J Surg 58B:426-435, 1976.

[24] Kember NF. Comparative patterns of cell division in epiphyseal cartilage plates in the rat. J Anat III:137-142, 1972.

[25] Juster M, Balmain-Oligo N. Sur la formation du squelette. II Croissance d'un os long. Metaphyse et virale périchondrale. Pathol Biol 19:33-42, 1971.

[26] Ham AW. Histology. 7th ed. Philadelphia: JB Lippincott, 1974.

[27] Gardner ED. The development and growth of bone and joint. J Bone J Surg Am 45A:856-862, 1963.

[28] Rivas R., Shapiro F. Structural stages in the development of the longs bones and epiphyses. A study in the New Zealand white rabbit. J Bone Joint Surg 59A:703-723, 2002.

[29] Langenskiold A. Edgren W. The growth mechanism of the epiphyseal cartilage in the light of the experimental observations. Acta Orthop Scand 19:19-24, 1949.

[30] Hunziker HB, Schenk RK. Quantitation of chondrocytes performance in growth plate during longitudinal bone growth. J Bone Joint Surg 69A:162-173, 1987.

[31] Ogden JA. Anatomy and Physiology of Skeletal Development in Skeletal Injury in the Child. Philadelphia: WB Saunders Co., pp. 23-63, 1990.

[32] Solomon L. Diametric growth of the epiphyseal plate. J Bone Joint Surg 48B:170-177, 1966.

[33] Shapiro F, Holtrop ME, Glimcher NJ. Organization and cellular biology of perichondrial ossification groove of Ranvier. J Bone Joint Surg 59A:703-723, 1977.

[34] Trevor D. Tarso-epiphyseal aclasis. A congenital error of epiphyseal development. J Bone Joint Surg 32B:204-213, 1950.

[35] Fairbank TJ. Dysplasia epiphysealis hemimelica (tarso epiphyseal aclasis). J Bone Joint Surg 237-242, 1956.

[36] Azouz EM, Slomic AM, Marton D, Rigault P, Finidori G. The variable manifestations of dysplasia epiphysealis hemimelica. Pediat Radiol 15:44-49, 1985. 